\title{
Computational contact mechanics
}

\author{
Peter Wriggers
}

Published online: 24 May 2012

(C) Springer-Verlag 2012

Within the last years, computational contact mechanics has been a topic of intense research. The aim of the development is to devise robust solution schemes and new discretization techniques that can be applied to different problem classes. These are wide ranging and include computational aspects of discretization techniques, solution algorithms for single- and multi-processor computing environments, multiscale approaches to contact problems, discrete element models for contact and multifield problems with contact constraints. Technical applications are in the area of interface problems for solid bodies, crash analysis, granular systems, failure processes in heterogeneous materials, textile and laminated composites, biomechanics as well as thermoelectro-mechanical contact problems. Due to the nature of the physical problems involved in contact mechanics the subject is multidisciplinary in which physicists, engineers and mathematicians have to work together in order to describe the complex phenomena at the contact interface. In this special edition different aspects of contact mechanics are considered that are related to the mathematical and mechanical foundation of solution methods and engineering applications.
This special issue is published within a series of special issues that celebrate the 25th anniversary of the journal Computational Mechanics. It contains some of the papers that were presented at the International Conference on Computational Contact Mechanics held in June 2011 at Leibniz Universität Hannover in Germany. The papers are concerned with the topics mentioned above. New discretization techniques for contact, like Mortar methods with dual Lagrange multipliers for 3D finite deformation contact are presented by B. Wohlmuth et al. Based on the XFEM methodology a three-dimensional contact discretization is developed by Mueller-Hoeppe et al. Special applications include the multiscale analysis of lubrication contact, see Budt et al., and biomechanical contact of hip joints, see A. Suwannachit et al. Furthermore contact within large granular systems is modeled by V. Visseq et al. and the contact of ropes is considered in the paper by D. Durville.

The wide spread spectrum of the papers provides the stateof-the art of modeling and simulation in computational contact mechanics.

Peter Wriggers, Hannover May 2012
P. Wriggers $(\bowtie)$

Institute of Continuum Mechanics,

30167 Hannover, Germany

e-mail: wriggers@ikm.uni-hannover.de 